\title{
Combination of Continuous Epidural Block and Rehabilitation in a Case of Complex Regional Pain Syndrome
}

\author{
Kazuhiro Hayashi ${ }^{1}$, Kimitoshi Nishiwaki ${ }^{2}$, Masato Kako ${ }^{1}$, Kentaro Suzuki ${ }^{1}$, \\ Keiko Hattori ${ }^{1}$, Koji Sato ${ }^{1}$, Izumi Kadono ${ }^{1,3}$ and Yoshihiro Nishida ${ }^{1,3}$ \\ ${ }^{1}$ Department of Rehabilitation, Nagoya University Hospital, Aichi, Japan \\ ${ }^{2}$ Department of Anesthesiology, Nagoya University Graduate School of Medicine, Aichi, Japan \\ ${ }^{3}$ Department of Orthopaedic Surgery, Nagoya University Graduate School and School of Medicine, Aichi, Japan
}

\begin{abstract}
Epidural analgesia is used to promote rehabilitation in patients with refractory complex regional pain syndrome (CRPS) who cannot bear physical programs due to intense pain. However, the actual rehabilitation process has not been focused in previous reports. Here, we outline our experience of treating a young woman with CRPS type 1 who underwent rehabilitation facilitated by a continuous lumbar epidural block.

A 15-year-old girl developed throbbing pain from her left toe to her ankle, with no obvious cause. She was admitted to the hospital 2 months after symptom onset for an assessment of pain intensity, range of motion, weight-bearing, neglect-like symptoms, pain catastrophizing, and a CRPS severe score with impaired activities of daily living. The rehabilitation program was initiated under facilitation of continuous epidural block. Her rehabilitation program included physical therapy, motor imagery, mirror therapy, and cognitive behavioral therapy. The intensity of the exercise was gradually increased without exacerbating her symptoms. Ultimately, she recovered completely after a continuous epidural block for 21 days and rehabilitation for 80 days.

A combination of continuous epidural block and intensive rehabilitation improved the symptoms of this patient. The treatment course would be helpful for planning rehabilitation programs in other patients with CRPS. (J Nippon Med Sch 2016; 83: 262-267)
\end{abstract}

Key words: catastrophizing, cognitive behavior therapy, complex regional pain syndrome, epidural anesthesia, physical therapy

\section{Introduction}

The clinical features of complex regional pain syndrome (CRPS) is not completely understood. CRPS is characterized by severe pain, sensory disturbances, allodynia, trophic changes, such as skin atrophy or altered hair and nail growth, localized osteoporotic changes, abnormal patterns of sweating, swelling and edema, changes in skin temperature, and reduced joint range of motion. In addition, there are movement abnormalities, such as weakness, tremor, dystonia, neglect-like symptoms, alterations in body image within the painful region, and/ or pain-related psychological disturbance ${ }^{1-6}$. The symptoms and their severity vary significantly between pa- tients.

A wide variety of treatment strategies for CRPS have also been reported, including pharmacologic therapy, psychological therapy, physical therapy, and interventional therapy, although the evidence base for the therapeutic benefits of each is still relatively sparse ${ }^{1-6}$. Patients' engagement with rehabilitation is often impaired by pain or other symptoms of CRPS. Intensive rehabilitation has been proposed for the initial treatment of $\mathrm{CRPS}^{7}$, but it is very painful. Epidural analgesia is recommended to promote rehabilitation in patients with refractory CRPS, after rehabilitation alone for 2-8 weeks ${ }^{7-10}$. However, previous reports of the use of continuous epidural analgesia in the

Correspondence to Kazuhiro Hayashi, PT, MSc, Department of Rehabilitation, Nagoya University Hospital, 65 Tsuruma-cho, Showa-ku, Nagoya, Aichi 466-8550, Japan

E-mail: hayashi.k@med.nagoya-u.ac.jp

Journal Website (http://www.nms.ac.jp/jnms/) 
management of CRPS have not focused on the rehabilitation $\operatorname{program}^{7-10}$, and information for this approach remains very limited. Here, we report the case of a young woman with CRPS type 1 who underwent rehabilitation facilitated by a continuous epidural block.

\section{Case Presentation}

\section{Patient History}

A 15-year-old female junior high school student had no medical history. She spontaneously developed pain radiating from the left toe to the ankle, without an obvious cause. Pain was throbbing and continuous from the onset of symptoms, and interfered with her sleep. She presented to a pain clinic 2 days after symptom onset. She was commenced on a course of oral acetaminophen $1,200 \mathrm{mg} /$ day and gabapentin $600 \mathrm{mg}$ /day in divided doses. Nonetheless, the intensity of the pain gradually increased, and ankle movement became weak and tremulous. Two months after the onset of pain, she reported piercing pain on movement and to light touch of the left foot, although there was no objective evidence of propagation of painful changes of the region. She was unable to tolerate bedclothes touching her left foot, could not wear a sock, and could not put that foot on the ground. She could not perform bathing, dressing, toilet use, transfers, or walk independently. Her activities of daily living (ADL) were substantially limited, and she was admitted to our hospital. On admission, she had allodynia, decreased skin temperature, skin color change, edema, excessive sweating, distortion of the body image of the painful region, limited range of motion, and absence of active movement of the entire left foot and ankle. On investigation, there were no trophic changes or decrease in sensation, and the rest of her physical examination, including $x$-ray findings, magnetic resonance imaging, and nerve conduction velocity studies, were normal.

Publication of this case was approved by the ethics committee of Nagoya University Hospital, Japan (No. 132), and the patient also provided informed consent.

\section{Examination}

Pain intensity was documented on a $100-\mathrm{mm}$ visual analog scale (VAS) $(0 \mathrm{~mm}=$ no pain, $100 \mathrm{~mm}=$ worst pain imaginable) and was used to obtain the mean pain intensity in day-to-day life. Active and passive plantar flexion and dorsiflexion of the left ankle were measured using a goniometer in knee flexion, within tolerable limits. Weight-bearing was measured using two calibrated scales. The patient stood with her feet shoulder-width apart, with one foot on each scale. While looking straight ahead, the patient was instructed to move slowly between "standing comfortably in a neutral position", "maintaining a half-squatting position" and "standing with as much weight as possible on your left leg". The patient was supervised, as necessary, to avoid falling and was permitted to hold on to parallel bars with her hands. Readings were taken within limits of pain tolerance.

Neglect-like symptoms (NLS) were assessed using the questionnaire developed by Frettlöh et $\mathrm{al}^{6}$, for which respondents use a VAS to indicate responses. The distance (mm) from the left end of the 100-mm line was defined as the NLS score. Consistent with Galer and Jensen ${ }^{11}$, motor neglect is considered a state requiring particular attention to move the affected limb, and cognitive neglect represents a reduced ability to perceive the presence of the affected limb. Both are viewed as subcategories of NLS, and each was included in two questions. Each score can range from 0 to 200.

The Pain Catastrophizing Scale (PCS) consists of 13 items ${ }^{12,13}$. Subjects rate how frequently they have experienced such thoughts/emotions ${ }^{12,13}$. The PCS is composed of three subscales: rumination (e.g., "I keep thinking about how much it hurts"), helplessness (e.g., "There is nothing I can do to reduce the intensity of the pain"), and magnification (e.g., "I wonder whether something serious may happen" $)^{12,13}$. The total score of the PCS can range from 0 to $52^{12,13}$.

ADLs were assessed using the Barthel Index, which evaluates 10 different abilities (feeding, transfer, mobility, dressing, stairs, toilet use, bathing, grooming, bladder, and bowel status) and gives a total score between 0 and 100 points $^{14}$.

The CRPS severity score consists of 17 items (eight items for the patient's subjective symptoms and nine items regarding the findings on physical examination) ${ }^{5}$. Each checked item counts as 1 point, and the sum of the scores constitutes the CRPS severity score ${ }^{5}$.

\section{Intervention and Outcome}

The patient was unable to tolerate even minimal rehabilitation on admission because of the absolute avoidance to touch due to extreme pain. A continuous lumbar epidural block was commenced immediately on admission $(0.15 \%$ ropivacaine, $4.0 \mathrm{~mL} / \mathrm{h})$, and oral drugs were discontinued. The visual analogue scale score for pain improved from 100 to 24, and the Barthel Index score for ADL improved from 10 to 60 after the epidural block had been instituted (Fig. 1). The patient reported no decrease in sensation or movement. Rehabilitation was commenced on the following day. At the first rehabilita- 
K. Hayashi, et al
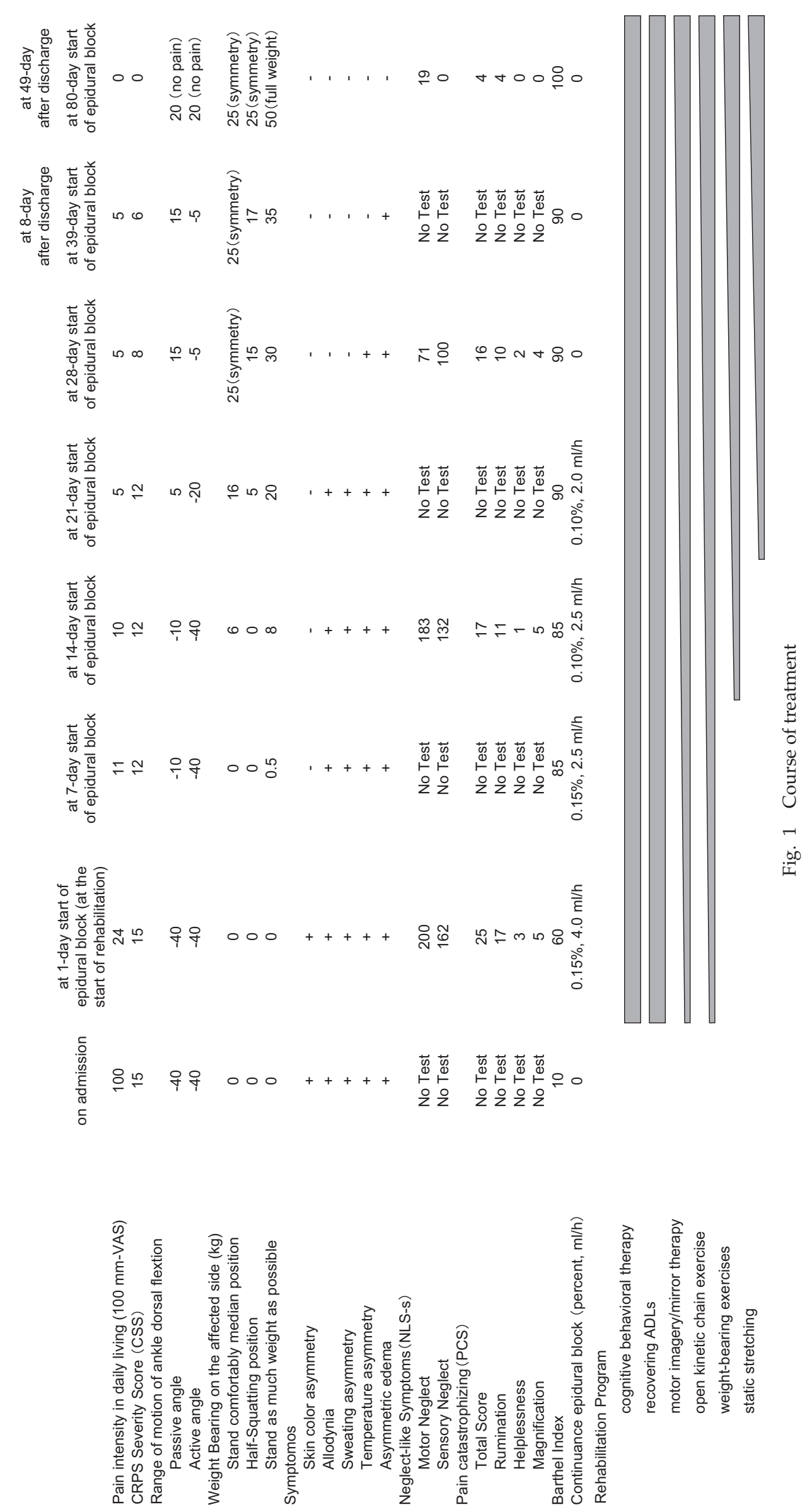
tion session, the patient was judged to have NLS and catastrophizing on the basis of her questionnaire responses. Pain-related states were also assessed; their subsequent course is shown below. The patient was also assessed for maladaptive cognition, including excessive fear of movement, a reactive attitude to medical treatment and excessive support from her mother. There were no other psychosocial risk factors for CRPS, such as school phobia or family problems ${ }^{15}$.

First, the patient and mother received education about CRPS, mechanisms of adaptive or maladaptive cognition for pain, and the importance of planning and pacing physical activity. Rehabilitation was mainly centered on recovering ADLs and avoiding too much focus on the painful foot. The presence of the physical therapists was gradually withdrawn to facilitate independence. The patient and physical therapist designed the program together.

The rehabilitation program in this case included physical therapy, motor imagery, mirror therapy, and cognitive behavioral therapy $(\mathrm{CBT})^{1-6}$. For motor imagery and mirror therapy, a mirror was placed vertically on the floor between the patients' legs. With the painful left limb hidden behind the mirror, the non-painful right limb was positioned so that its reflection was superimposed on the painful one. This strategy first introduced visual recognition of limb laterality, moved on to imagined limb movements and culminated in mirror movements ${ }^{16,17}$. The patient was instructed to perform non-painful right limb exercises from the toe to the knee, while observing the reflected image. The ranges and speeds of these exercises were dictated by pain intensity ${ }^{18}$. The maximum duration of each motor imagery and mirror therapy session was 10 minutes. Moreover, the patient was encouraged to replace the maladaptive cognition for pain with a more adaptive one in CBT. The patient was educated in coping style, and encouraged to continue being exposed to more activities through the study. Open kinetic chain exercises were also started in the left foot in flexion-extension, varus-valgus, and adduction-abduction. Weight-bearing exercises began with the patient putting her left foot on the ground in a standing position using the parallel bars, and additional force was applied gradually.

As rehabilitation began, the patient was observed to have less fear of movement and recovered active movement, albeit with a tremor. Subsequently, the intensity of the exercise was gradually increased based on ability, and the continuous epidural block was gradually weaned.
Seven days after the initiation of epidural anesthesia, the patient could put her left foot on the ground. Her confidence to engage in activities had improved so that gait exercises with a pair of crutches could be initiated in the hospital ward. Skin color, asymmetry and hyperpathia to pinprick had also improved. Two weeks after initiation of treatment, the patient could squat using the parallel bars and bear a little weight on her painful left limb. At three weeks, the patient voluntarily proposed an activity program that included static stretching of that ankle and foot. After 21 days of treatment, the epidural infusion was terminated, but the pain was not markedly increased. There were no signs of infection throughout the epidural infusion. The patient was then able to leave the hospital for a day. After 28 days, allodynia, body image, and sweating asymmetry had improved. The patient was discharged from the hospital after 31 days, taking pregabalin $225 \mathrm{mg} /$ day orally in divided doses. By 39 days from the initiation of treatment, the patient had returned to school with crutches. Skin temperature asymmetry had improved. After 66 days, the patient attended school without crutches, and by 80 days, formal rehabilitation was completed, when all the symptoms of CRPS had resolved. The patient was encouraged to continue engaging in more activities in everyday life after completion of the treatment sessions.

\section{Discussion}

This patient with CRPS responded well to combination therapy of continuous epidural infusion and rehabilitation. Her symptoms resolved gradually, and she was able to resume daily life without pain.

Rehabilitation is recommended for $\mathrm{CRPS}^{1-6}$, but the optimum rehabilitation strategy and intensity of exercise have not been identified in a clinical trial. The primary benefit of combining interventional therapy and rehabilitation is the ability to control the pain intensity and promote the rehabilitation program ${ }^{1}$. Because certain cases of CRPS resolve under pharmacotherapy and rehabilitation alone, epidural analgesia is usually initiated after an observation period of $2-8$ weeks $^{7-10}$. The duration of continuous administration was 14 days in most cases, but it varied from several days to 12 weeks ${ }^{19}$. This strategy was effective for our patient. Having partial attenuation of extreme pain, the intensity of the exercise was gradually increased without deterioration in CRPS symptoms. Careful monitoring of a variety of symptoms provided information for decisions about rehabilitation and, ultimately, when to stop the epidural infusion. 
The rehabilitation program in this case included physical therapy, motor imagery, mirror therapy, and CBT. Physical therapy is reported to improve physical function and performance and is considered the cornerstone and first line treatment for $\mathrm{CRPS}^{7,20}$. Our approach encouraged exposure to fearful activities, as the patient was given confidence by the presence of epidural analgesia. Graded exposure to the fearful activities improves pain and painrelated psychological disturbance in $\mathrm{CRPS}^{21}$. Motor imagery and mirror therapy are effective means of reconciling dynamic central mismatch, improving activation of motor networks and reducing pain and stiffness in early CRPS, but are reportedly not effective in chronic CRPS ${ }^{18,22}$. This case was classified as early stage CRPS, as the symptoms had begun within 3 months of diagnosis ${ }^{2}$.

Rehabilitation in this case was combined with CBT, which is also reported to have a beneficial therapeutic effect in CRPS ${ }^{23,24}$. CBT is an effective treatment for chronic pain, achieving reductions in pain and pain-related interference with ADLs, improvements in mood and coping, and reductions in negative beliefs and cognitions, such as catastrophizing ${ }^{25}$. The technique traditionally includes coping skills training, cognitive restructuring, pacing, distraction, goal setting, and homework adherence ${ }^{25}$.

Our patient was an adolescent, so our experiences may not be generalizable to adults. Indeed, the symptoms of CRPS and the response to treatment likely differ between children and adults ${ }^{7,26}$. In children, rehabilitation induces remission of symptoms in the vast majority ${ }^{7}$. Meanwhile, adults more frequently have significant trauma preceding their CRPS, and perhaps, it is less of a psychopathophysiologic phenomenon 7 . It could be argued that our rehabilitation program was relatively conservative and could have been even more aggressive; however, we prioritized avoiding over-exertion and its potential to exacerbate pain, the fear of movement and other symptoms of CRPS. Clinical trials are needed to establish the treatment efficacy of a rehabilitation program facilitated by epidural analgesia in CRPS.

\section{Conclusions}

Combination therapy with a continuous epidural block and rehabilitation improved CRPS symptoms in this case. This case report outlined, for the first time, the rehabilitation course in the management of CRPS.

Conflict of Interest: The authors certify that no affiliation or financial involvement exists between them and any organization with a direct interest in the subject matter or materials discussed in the article.

\section{References}

1. Harden RN, Oaklander AL, Burton AW, Perez RS, Richardson K, Swan M, Barthel J, Costa B, Graciosa JR: Complex regional pain syndrome: practical diagnostic and treatment guidelines, 4th edition. Pain Med 2013; 14: 180229.

2. Harden RN, Bruehl SP: Complex Regional Pain Syndrome. In Bonica's Management of Pain (Fishman SM, Ballantyne JC, Rathmell JP, eds), 4th ed. 2009; pp 314-330, Lea \& Febiger, Philadelphia, PA, USA.

3. O'Connell NE, Wand BM, McAuley J, Marston L, Moseley GL: Interventions for treating pain and disability in adults with complex regional pain syndrome. Cochrane Database Syst Rev 2013; 30: CD009416.

4. Tran DQ, Duong S, Bertini P, Finlayson RJ: Treatment of complex regional pain syndrome: a review of the evidence. Can J Anaesth 2010; 57: 149-166.

5. Harden RN, Bruehl S, Perez RS, Birklein F, Marinus J, Maihofner C, Lubenow T, Buvanendran A, Mackey S, Graciosa J, Mogilevski M, Ramsden C, Schlereth T, Chont M, Vatine JJ: Development of a severity score for CRPS. Pain 2010; 151: 870-876.

6. Frettlöh J, Hüppe M, Maier C: Severity and specificity of neglect-like symptoms in patients with complex regional pain syndrome (CRPS) compared to chronic limb pain of other origins. Pain 2006; 124: 184-189.

7. Sherry DD, Wallace CA, Kelley C, Kidder M, Sapp L: Short- and long-term outcomes of children with complex regional pain syndrome type I treated with exercise therapy. Clin J Pain 1999; 15: 218-223.

8. Farid IS, Heiner EJ: Intrathecal local anesthetic infusion as a treatment for complex regional pain syndrome in a child. Anesth Analg 2007; 104: 1078-1080.

9. Saito Y, Baba S, Takahashi A, Sone D, Akashi N, Koichihara R, Ishiyama A, Saito T, Komaki H, Nakagawa E, Sugai K, Sasaki M, Otsuki T: Complex regional pain syndrome in a 15-year-old girl successfully treated with continuous epidural anesthesia. Brain Dev 2015; 37: 175-178.

10. Cooper DE, DeLee JC, Ramamurthy S: Reflex sympathetic dystrophy of the knee. Treatment using continuous epidural anesthesia. J Bone Joint Surg Am 1989; 71: 365369.

11. Galer BS, Jensen M: Neglect-like symptoms in complex regional pain syndrome: Results of a self-administered survey. J Pain Symptom Manage 1999; 18: 213-217.

12. Sullivan MJL, Bishop SR, Pivik J: The Pain Catastrophizing Scale: development and validation. Psychol Assess 1995; 7: 524-532.

13. Matsuoka H, Sakano Y: Assessment of cognitive aspect of pain: development, reliability, and validation of Japanese version of Pain Catastrophizing Scale. Jpn J Psychosom Med 2007; 47: 95-102.

14. Mahoney FI, Barthel DW: Functional evaluation: the Barthel Index. Md State Med J 1965; 14: 61-65.

15. Sherry DD, Weisman R: Psychologic aspects of childhood reflex neurovascular dystrophy. Pediatrics. 1988; 81: 572578.

16. Bowering $\mathrm{KJ}, \mathrm{O}^{\prime}$ Connell NE, Tabor A, Catley MJ, Leake HB, Moseley GL, Stanton TR: The effects of graded motor imagery and its components on chronic pain: a systematic review and meta-analysis. J Pain 2013; 14: 3-13.

17. Moseley GL: Graded motor imagery is effective for longstanding complex regional pain syndrome: a randomised 
controlled trial. Pain 2004; 108: 192-198.

18. Ezendam D, Bongers RM, Jannink MJ: Systematic review of the effectiveness of mirror therapy in upper extremity function. Disabil Rehabil 2009; 31: 2135-2149.

19. Moufawad S, Malak O, Mekhail NA: Epidural infusion of opiates and local anesthetics for Complex Regional Pain Syndrome. Pain Pract 2002; 2: 81-86.

20. Rho RH, Brewer RP, Lamer TJ, Wilson PR: Complex regional pain syndrome. Mayo Clin Proc 2002; 77: 174-180.

21. de Jong JR, Vlaeyen JW, Onghena P, Cuypers C, den Hollander M, Ruijgrok J: Reduction of pain-related fear in complex regional pain syndrome type I: the application of graded exposure in vivo. Pain 2005; 116: 264-275.

22. McCabe CS, Haigh RC, Ring EF, Halligan PW, Wall PD, Blake DR: A controlled pilot study of the utility of mirror visual feedback in the treatment of complex regional pain syndrome (type 1). Rheumatology (Oxford) 2003; 42: 97101.

23. Vladimir Tichelaar YI, Geertzen JH, Keizer D, Paul van, Wilgen C: Mirror box therapy added to cognitive behav- ioural therapy in three chronic complex regional pain syndrome type I patients: a pilot study. Int J Rehabil Res 2007; 30: 181-188.

24. Lee BH, Scharff L, Sethna NF, McCarthy CF, ScottSutherland J, Shea AM, Sullivan P, Meier P, Zurakowski D, Masek BJ, Berde CB: Physical therapy and cognitivebehavioral treatment for complex regional pain syndromes. J Pediatr 2002; 141: 135-140.

25. Buenaver LF, Campbell CM, Haythornthwaite JA: Cognitive-Behavioral Therapy for Chronic Pain. In Bonica's Management of Pain (Fishman SM, Ballantyne JC, Rathmell JP, eds), 4th ed. 2009; pp 1220-1229, Lea \& Febiger, Philadelphia, PA, USA.

26. Bernstein BH, Singsen BH, Kent JT, Kornreich H, King K, Hicks R, Hanson V: Reflex neurovascular dystrophy in childhood. J Pediatr 1978; 93: 211-215.

(Received, February 8, 2016)

(Accepted, September 26, 2016) 